\title{
Mutation of the RDR1 gene caused genome-wide changes in gene expression, regional variation in small RNA clusters and localized alteration in DNA methylation in rice
}

Ningning Wang ${ }^{1,2,5+}$, Di Zhang ${ }^{1 \dagger}$, Zhenhui Wang ${ }^{1}$, Hongwei Xun ${ }^{1}$, Jian Ma², Hui Wang ${ }^{1}$, Wei Huang ${ }^{1}$, Ying Liu', Xiuyun Lin ${ }^{3}$, Ning Li ${ }^{1}$, Xiufang Ou', Chunyu Zhang ${ }^{1,4}$, Ming-Bo Wang ${ }^{5}$ and Bao Liu' ${ }^{1 *}$

\begin{abstract}
Background: Endogenous small (sm) RNAs (primarily si- and miRNAs) are important trans/cis-acting regulators involved in diverse cellular functions. In plants, the RNA-dependent RNA polymerases (RDRs) are essential for smRNA biogenesis. It has been established that RDR2 is involved in the $24 \mathrm{nt}$ siRNA-dependent RNA-directed DNA methylation (RdDM) pathway. Recent studies have suggested that RDR1 is involved in a second RdDM pathway that relies mostly on $21 \mathrm{nt}$ smRNAs and functions to silence a subset of genomic loci that are usually refractory to the normal RdDM pathway in Arabidopsis. Whether and to what extent the homologs of RDR1 may have similar functions in other plants remained unknown.

Results: We characterized a loss-of-function mutant (Osrdr1) of the OsRDR1 gene in rice (Oryza sativa L.) derived from a retrotransposon Tos 17 insertion. Microarray analysis identified 1,175 differentially expressed genes (5.2\% of all expressed genes in the shoot-tip tissue of rice) between Osrdr1 and WT, of which 896 and 279 genes were up- and down-regulated, respectively, in Osrdr1. smRNA sequencing revealed regional alterations in smRNA clusters across the rice genome. Some of the regions with altered smRNA clusters were associated with changes in DNA methylation. In addition, altered expression of several miRNAs was detected in Osrdr1, and at least some of which were associated with altered expression of predicted miRNA target genes. Despite these changes, no phenotypic difference was identified in Osrdr1 relative to WT under normal condition; however, ephemeral phenotypic fluctuations occurred under some abiotic stress conditions.

Conclusions: Our results showed that OsRDR1 plays a role in regulating a substantial number of endogenous genes with diverse functions in rice through smRNA-mediated pathways involving DNA methylation, and which participates in abiotic stress response.
\end{abstract}

Keywords: Gene expression, Epigenetics, Small RNA, DNA methylation, RDR1, Oryza sativa L

\footnotetext{
* Correspondence: Baoliu@nenu.edu.cn

${ }^{\dagger}$ Equal contributors

'Key Laboratory of Molecular Epigenetics of Ministry of Education (MOE),

Northeast Normal University, Changchun 130024, China

Full list of author information is available at the end of the article
} 


\section{Background}

RNA silencing is an evolutionally conserved gene regulation mechanism in eukaryotes mediated by $20-25 \mathrm{nt}$ noncoding small $(\mathrm{sm})$ RNAs. These smRNAs are processed from double-stranded (ds) or hairpin RNA molecules by Dicer-like (DCL) proteins, and guide RNA-induced silencing complexes to cognate single-stranded RNAs based on sequence complementarity, and result in degradation of the targeted RNAs [1-3]. In plants, there are several different classes of smRNAs, including 20-24 nt micro RNAs (miRNAs) processed by DCL1, 21-22 nt small interfering RNAs (siRNAs) by DCL4 and DCL2, and the $24 \mathrm{nt}$ heterochromatin-associated siRNAs by DCL3. miRNAs play an important role in plant development by directing posttranscriptional gene silencing (PTGS) of regulatory genes such as those encoding transcription factors. Similarly, 21-22 nt siRNAs guide the degradation of viral RNAs as well as some endogenous mRNAs and are important for plant defense against viruses and for some aspects of plant development [4-6]. Unlike these PTGSassociated smRNAs, the $24 \mathrm{nt}$ siRNAs are associated with RNA-directed DNA methylation (RdDM), a plantspecific de novo DNA methylation pathway required for transcriptional silencing of transposable elements and other DNA repeats to maintain genome stability [7-10].

The biogenesis of siRNAs in plants requires the activity of RNA-dependent RNA polymerase (RDR), which converts single-stranded RNAs to dsRNA precursors of siRNAs. The dicot model plant Arabidopsis thaliana has six $R D R$ genes, i.e., RDR1, RDR2, RDR3a, RDR3b, $R D R 3 c$ and RDR6 [11], of which three RDRs (RDR1, $R D R 2$ and $R D R 6$ ) are shown to play roles in the RNA silencing pathways. $R D R 2$ is required for 24 nt siRNA biogenesis and therefore involved in the canonical RdDM pathway [7-9]. RDR6 is involved in the production of the endogenous 21 nt trans-acting siRNAs and also essential for sense transgene-induced PTGS $[12,13]$. Both RDR6 and RDR2 are also involved in viral siRNA accumulation in infected Arabidopsis plants [14-16]. The function of RDR1 in RNA silencing is less understood, but recent studies have shown that it is involved in siRNA biogenesis from a subset of RNA viruses [17-19]. Furthermore, $r d r 1$ mutant of Arabidopsis showed loss of DNA methylation in a subset of genomic loci in comparison to wild-type Arabidopsis plants [20,21], suggesting that $R D R 1$ plays a role in the recently identified non-canonical, 21 nt siRNA-directed RdDM pathway $[20,21]$. However, the function of $R D R 1$ in gene regulation from a genome-wide perspective has not been investigated in any plant.

In contrast to Arabidopsis that has six RDR genes, the RDR family of rice (Oryza sativa L.), a model plant for monocots, contains only three members, namely OsRDR1, OsRDR2 and OsRDR6 [11,22,23]. A previous study showed that OsRDR1 has a similar function to its counterparts in Arabidopsis and tobacco (Nicotiana tabacum) in PTGSbased silencing of certain RNA viruses, such as Bromovirus $[6,19,24]$. To investigate if OsRDR1 plays a role in regulation of endogenous genes in rice, we characterized a lossof-function mutant of OsRDR1 derived from a disruptive LTR retrotransposon (Tos17) insertion into the 2nd exon of the gene. We investigated genome-wide changes in gene expression and smRNA profiles, localized changes in DNA methylation, and phenotypes under normal and several abiotic stress conditions in this rice $r d r 1$ mutant.

\section{Results}

\section{Characterization of the rice RDR1 mutant (Osrdr1)}

We obtained a LTR retrotransposon Tos17 [25] insertion line for OsRDR1 (accession number H0643) from the Tos17 insertion mutant library of rice cv. Hitomebore (www.cns.fr/spip/Oryza-sativa-retrotransposon-Tos17.html). Molecular characterization identified H0643 as heterozygous for a Tos 17 insertion into the second exon of OsRDR1 (Figure 1a). We obtained the homozygous mutant (OsRDR1-/- or Osrdr1) and its sibling wild type (WT) plants by selfing of the heterozygous plant (OsRDR1+/-) for five successive generations. In each generation, the three kinds of genotypes, WT, heterozygote and homozygous mutant, were selected based on locus-specific PCR amplifications (Figure 1a). Both the heterozygous and homozygous plants for OsRDR1 showed no discernibly altered phenotypes in the entire growth and developmental period over multiple generations under normal field conditions (Figure 1b). Semi-quantitative and real-time quantitative (q)RT-PCR analyses confirmed that the homozygous OsRDR1 mutant (Osrdr1) had a complete loss of OsRDR1 expression in shoot-tip tissue wherein the gene was highly expressed in WT plants (Figure 1c). This indicated that the exonic insertion of Tos 17 knocked out the expression of OsRDR1, and hence, abolished its function.

\section{Genome-wide changes in gene expression in Osrdr1}

We profiled the transcriptome of shoot-tip tissues between Osrdr1 and its sibling WT plants using the Affymetrix GeneChip Rice ${ }^{\circ}$ Genome Array (The Affymetrix, Inc. Santa Clara, CA, USA). After normalization of the microarray data, we detected 57,381 expressed genes in the shoot-tip tissue of rice. The expression levels of 22,419 genes were conserved between Osrdr1 and WT, but 896 and 279 genes showed significant up- and down-regulation in Osrdr1, respectively (Figure 2a). A Gene Ontology (GO) category analysis of these 1,175 differentially expressed genes showed that they were enriched in a variety of GO categories (Figure 2b). However, these 1,175 differentially expressed genes were found to distribute non-randomly across the 12 rice chromosomes $(\mathrm{P}=2.2 \mathrm{E}-16$, based on Chi square test). For example, chromosomes 1, 2 and 3 
(a) Os02g0736200/LOC_Os02g50330

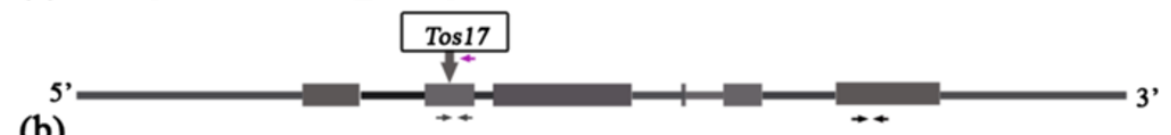

(b)
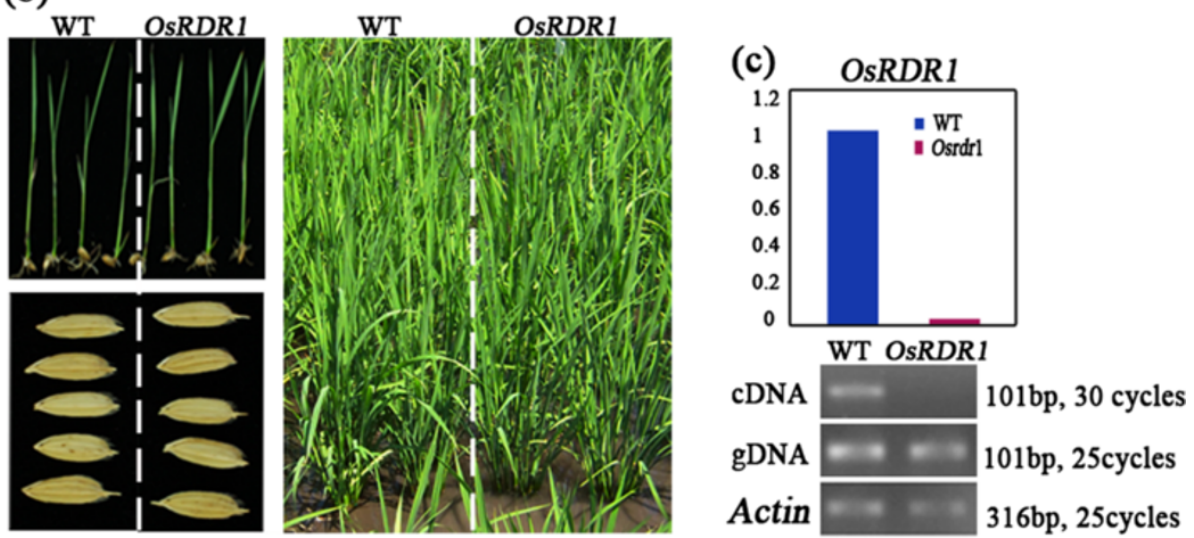

Figure 1 OsRDR1 gene expression is abolished in the Tos 17 insertion mutant Osrdr1. (a) Structure of the OsRDR1 locus with the Tos 17 insertion into the 2 nd exon (vertical arrows). Heterozygous/homozygous individuals were selected based on PCR which primers were indicated by the purple arrows while primers for OsRDR1 expression analysis were indicated by black arrows. (b) Germinated seedling, paddy-field-grown plant and kernel phenotypes of wide-type (WT) and Osrdr1. (c) Relative to WT, OsRDR1 expression was silenced in shoots of Osrdr1, as evidenced by both qRT-PCR (top) and semi-quantitative RT-PCR (bottom) amplifications with gene-specific primers downstream of the Tos 17 insertion (horizontal arrows). Genomic DNAs were used as positive controls.

contained significantly more distributions than the rest chromosomes (Figure 2c). It is also clear from the data that within a given chromosome, the distribution is also nonrandom, for example, the distributions are almost exclusively confined to the long arms of chromosomes 4 and 8 and 9 relative to their respective short-arms (Figure 2c).

The highly reproducible microarray profiles among three biological replicates for both Osrdr1 and its WT sibling plants testified reliability of the data and their analysis. All microarray data have been submitted to the GEO repository under the accession number of GSE58007. To further verify the quality of the microarray data and analysis, we analyzed 18 genes representing both upand down-regulation in Osrdr1 vs. WT, as well as equal expression between the two lines using qRT-PCR assay on the same cDNAs as used for microarray. The qRTPCR results were highly consistent with the microarray data for almost all the 18 tested genes in levels or at least in trends of expression changes (Figure 2d), confirming reliability of the microarray analysis.

\section{Alteration in smRNA clusters in Osrdr1}

Previous studies have established that $R D R 1$ function is required for biogenesis and/or amplification of some types of RNA virus-related smRNA accumulation in Arabidopsis [26] and tobacco (Nicotiana tabacum) [27]. These findings promoted us to test whether loss of function of OsRDR1 may have a general impact on "normal" smRNA abundance in rice, and we investigated this issue by high-throughput smRNA sequencing. Comparison of the 10,398,592 clean smRNA reads from Osrdr1 with the 9,339,435 reads from its sibling WT (see Methods) revealed highly similar profiles in both size distributions and sequence categories of the smRNAs between Osrdr1 and WT (Figure 3a, b), suggesting that the overall smRNA abundance was not generally affected by the loss of function of OsRDR1.

Genome-wide overall similarity in smRNA abundance does not necessitates absence of smRNA fluctuations in localized smRNA clusters, because up- and downregulated smRNA accumulation can be masked by reciprocal compensation. We thus investigated localized smRNA accumulation between Osrdr1 and its sibling WT by mapping the cleaned smRNA reads to $100 \mathrm{bp}$ sliding windows (being reflected as smRNA clusters) across each of the 12 rice chromosomes, normalized the reads to Reads per Million (RPM), and then compared the RPM smRNA clusters between Osrdr1 and WT. Using 4-fold difference as a cut-off threshold, we identified many smRNA clusters with differential abundance between Osrdr1 and its sibling WT, which were uniformly distributed across the entire length of each chromosome (Figure 3c). Next, we extracted the differentially expressed smRNAs between Osrdr1 and WT (also based on a cut-off threshold of 4-fold difference) in the size range of 20-24 nt, which should parsimoniously contain all siRNAs, and mapped them to the same $100 \mathrm{bp}$ windows across each chromosome. We found that this subset of differential 


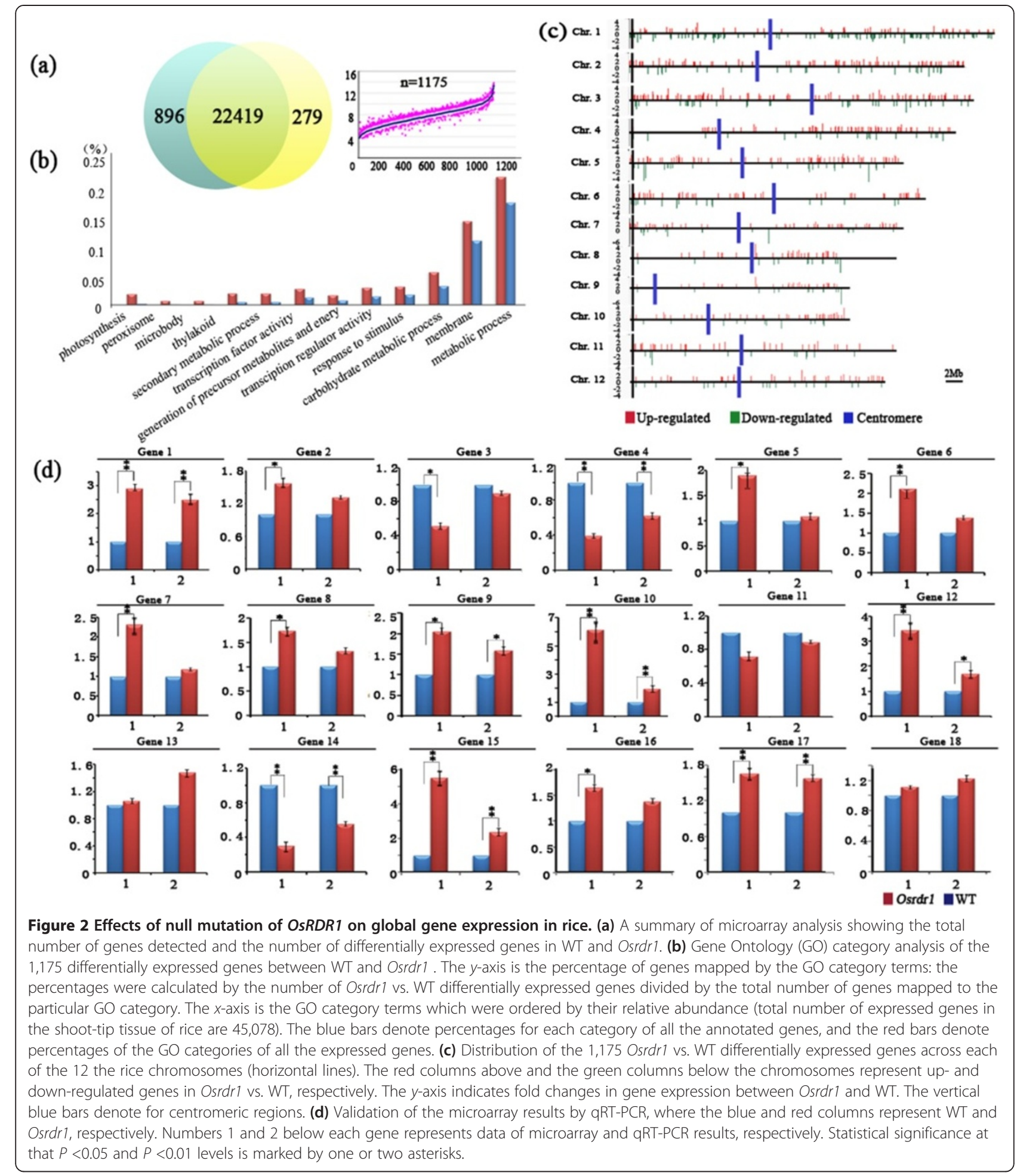

smRNA clusters also distribute on both arms of each chromosome (Additional file 1), although due to their smaller numbers, we cannot rule out the possibility that the distribution might show "hot spots" within a given chromosome. Taken together, the smRNA sequencing data suggested that loss-of-function mutation in OsRDR1 caused extensive alterations in smRNA clusters across each chromosome and throughout the genome, but it did not result in marked fluctuations of overall smRNA profiles, probably due to more or less equally increased and decreased abundance of the smRNA clusters which offset each other. 


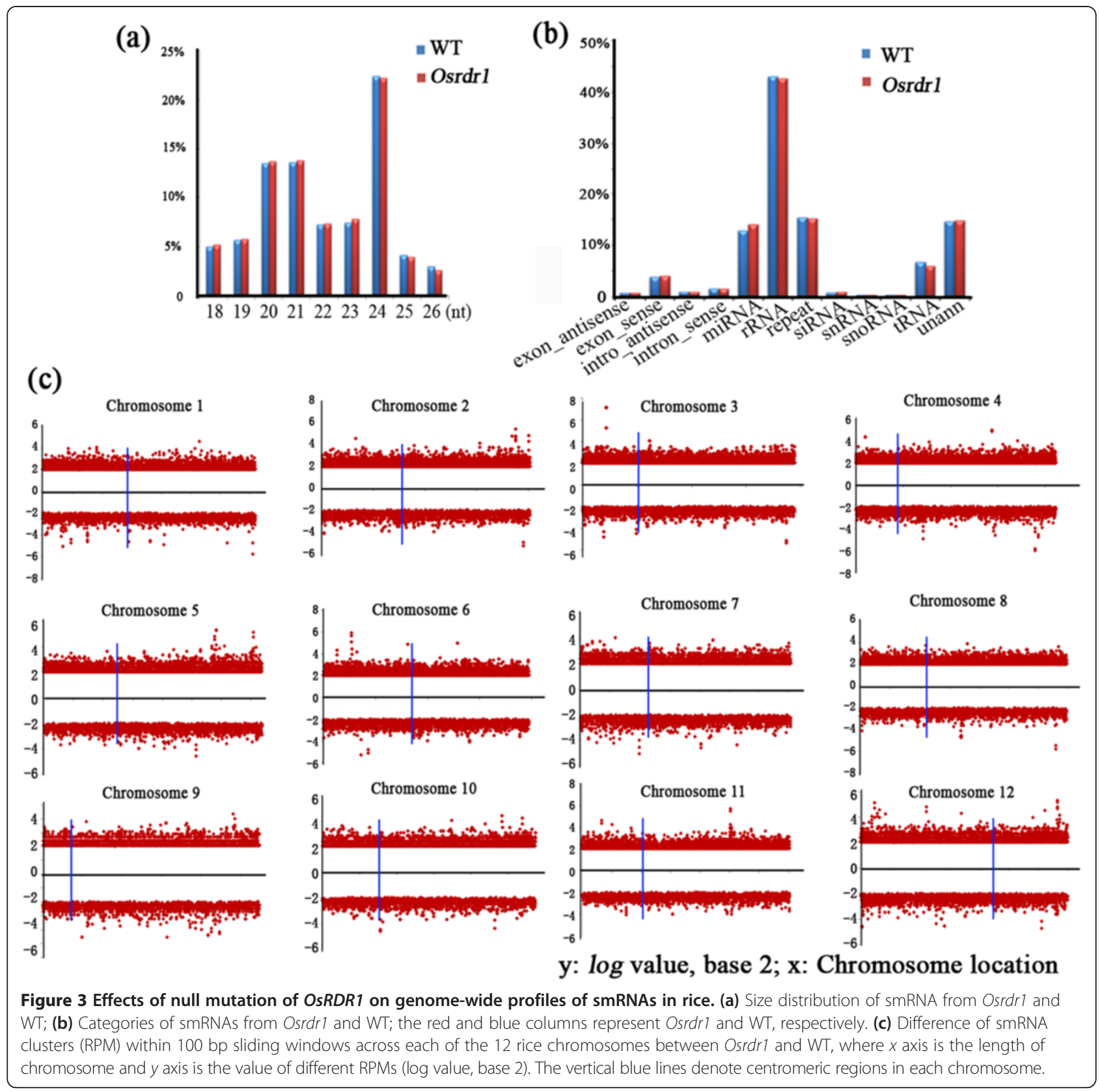

Altered expression of miRNAs and their target genes in Osrdr1

Given the diverse important roles played by miRNAs, we investigated if their accumulation might be affected in the Osrdr1 mutant. Previous computational and cloning studies have identified ca. 300 miRNAs from 86 miRNA families in rice $[28,29]$. Based on this information, we first analyzed the abundance of known rice miRNAs (OsamiRNAs) (listed in miRBase14.0) in Osrdr1 and WT. This analysis (Figure 4a, b; Additional file 2) indicated that: (1) majority (90.7\%) of the known Osa-miRNAs were expressed equally or nearly so between $\operatorname{Osrdr} 1$ and WT; (2) some miRNAs (5\%) showed $>2$ fold increased expression in Osrdr1 relative to WT, with the highest expression ratio reaching 9.0:1 ( $t$-test, $\mathrm{P}<0.05)$; (3) some miRNAs $(4.3 \%)$ showed $>2$ fold decrease in expression in Osrdr1 relative to WT, with the lowest expression ratio of 1:6.1 observed for miR167j between mutant and WT ( $t$-test, $\mathrm{P}<0.05$ ); (4) Osa-miR395p and Osa-miR395s, being from the same miRNA family, showed changes in expression to opposite directions, with an expression ratio of 1:5.6 for osa-miR395p but 4.2:1 for osa-miR395s in mutant vs. WT $(t$-test, $\mathrm{P}<0.05)$. To verify the expression differences based on the smRNA sequencing data, we performed semi-nested qRT-PCR analysis of four miRNAs in mutant and WT. The qRT-PCR results were found 


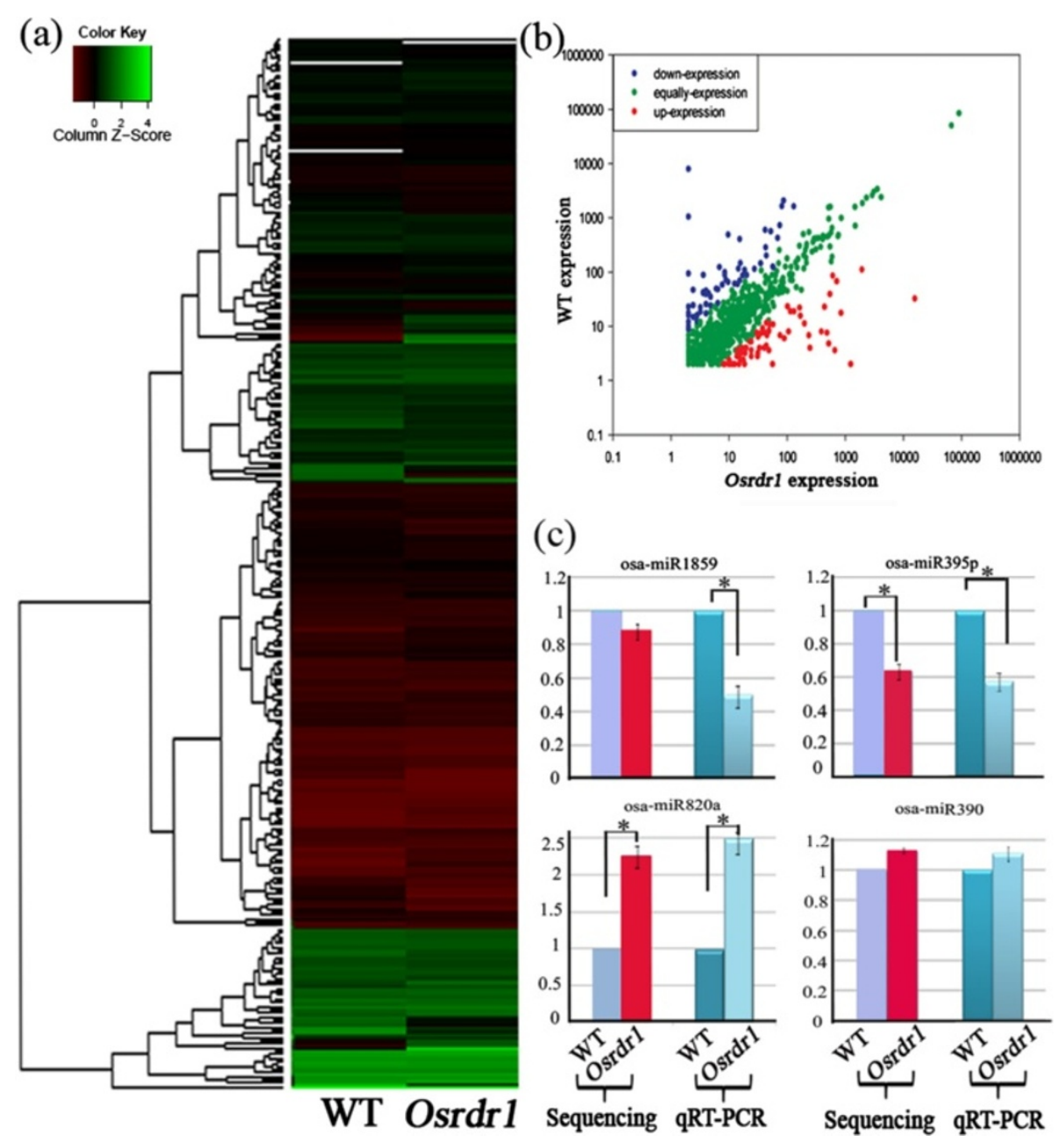

Figure 4 Effects of null mutation of OsRDR1 on abundances of miRNAs in rice. (a) Expression comparison of known miRNAs between Osrdr1 and WT. (b) Three patterns of expression changes of known miRNAs. The red and blue spots represent up and down-regulated in Osrdr1, respectively; the green spots represent no variation between Osrdr1 and WT. (c) qRT-PCR to verify variation of miRNA accumulation between Osrdr1 and WT. Statistical significance is marked by asterisks.

consistent with the smRNA sequencing data (Figure 4c), confirming the changes in miRNA expression between Osrdr1 and WT.

In addition to the known miRNAs, we identified a total of 10 putative novel osa-miRNAs from the smRNA data of the mutant and WT plants based on prediction of pre-miRNA-like stem-loop structures in sequences surrounding the smRNA sequences in the rice genome (Additional file 3). Three of these novel miRNAs (OsrmiRNA-N5.1, -N5.2 and -N5.3) had an identical mature sequence but corresponded to three independent genomic loci, thereby forming a novel miRNA family. For the putative miRNA Osr-miRNA-N7, smRNA reads were detected from both the 5' (5p) and the 3' (3p) half of the predicted stem-loop structure, but the 5p smRNA showed a higher abundance than the 3p smRNA (Additional file 3), indicating that the 5p smRNA is the guide strand (miRNA) whereas the 3p smRNA is the passenger strand (miRNA*) [30]. Like the known miRNAs, these novel miRNAs also showed expression variation between Osrdr1 and WT, with 3 showing expression only in Osrdr1, and 5 showing expression only in WT plants (Additional file 3). Taken together, our results suggest that OsRDR1 was likely involved in miRNA accumulation in rice.

To investigate if the altered miRNA accumulation in Osrdr1 relative to WT was associated with changes in miRNA target gene expression, we compared the miRNA expression profiles (abundance) derived from the smRNA sequencing data with the target gene expression levels based on the microarray data. We did not find a generalized relationship between the miRNA abundance and target gene expression levels (Additional file 4a). Instead, four types of relationships were recognized for a subset of miRNAs and their predicted targets (Additional file 4b), 
which included: (1) reduced miRNA abundance was correlated with up-regulated expression of target genes in Osrdr1 relative to WT (Additional file 4b-i); (2) increased miRNA abundance was correlated with downregulated expression of target genes in Osrdr1 relative to WT (Additional file 4b-ii); (3) both miRNAs and their target genes were up-regulated in Osrdr1 relative to WT (Additional file 4b-iii); (4) both miRNAs and their target genes were down-regulated in Osrdr1 relative to WT (Additional file 4b-iv). The first two types of relationships supported a role of miRNAs in downregulating expression of their predicted target genes. The last two types of relationships could be a result of concordant transcriptional regulation of the miRNAs and their target genes caused by another more upstream regulator(s) whose expression or activity was modified due to loss of function of OsRDR1. All small RNA data have been submitted to GenBank under the accession numbers of SRP042238.

\section{Locus-specific alteration of DNA methylation in Osrdr1}

As the Arabidopsis RDR1 has been shown to play a role in the non-canonical, NERD-dependent RdDM pathway $[20,21]$, we were interested to know if OsRDR1 might have a similar function in rice. We therefore examined cytosine methylation and gene expression levels of 10 selected genomic loci in Osrdr1 and its sibling WT using bisulphite sequencing and qRT-PCR analysis. These loci overlapped with two transposable elements (TEs) and three protein-coding genes, which were chosen as representatives because they all showed alteration in smRNA clusters in Osrdr1 relative to WT (Additional file 5). The bisulphite-sequenced regions for the two TEs (retrotransposon Tos 17 and DNA transposon Pong) included: (1) portions of the 5'- and 3'-LTRs together with their immediate flanking regions of two Tos17 copies (located on chromosomes 10 and 7 , respectively) (Additional file 5a, b); (2) the 5' termini along with their immediate flanking regions of two Pong copies (located on chromosomes 2 and 9, respectively) (Additional file 5c, d), and; (3) a body-region of the transposase-encoding ORF of Pong (Additional file 5e) that is shared by all conserved copies of the element. The bisulphite-sequenced regions of the three protein-coding genes are all within their 5 '-upstream regions (Additional file 5f, g, h).

The bisulphite sequencing results showed that: (1) of the five Tos 17 regions analyzed, only the 5' LTR region for the Tos 17 copy located on chromosome 7 showed marked decrease (by ca. 30\%) in CG and CHG methylation but not in $\mathrm{CHH}$ methylation in Osrdr1 relative to WT (Figure 5a and Additional file 5b); (2) for the three Pong regions analyzed, only the 5'region of the copy located on chromosome 2 showed clear methylation changes: decrease in CG methylation by $20 \%$ and increase in both $\mathrm{CHG}$ and $\mathrm{CHH}$ methylation by approximately $30 \%$ and $50 \%$, respectively, in Osrdr1 relative to WT (Figure 5a and Additional file 5c); of the three genic loci analyzed, only one (Os06g0316000) showed increase in CG methylation by $c a$. $50 \%$ in Osrdr1 relative to WT, while methylation of the other two regions were unchanged (Figure 5a and Additional file 5f).

The two TEs showed significant up-regulation in Osrdr1 relative to WT (Figure 5b), consistent with a decrease in methylation at the 5' regions of one copy of each TEs in Osrdr1 (Figure 5a). Notably, all the three genes analyzed did not show the expected relationship between DNA methylation state of their 5'-regulatory regions and expression levels. Specifically, one gene (Os06g0316000) that showed increase in CG methylation in Osrdr1 was upregulated in expression (Figure $5 \mathrm{~b}$ ); the remaining two genes showed down-regulation in Osrdr1 relative to WT despite the lack of methylation changes in the bisulphitesequenced regions (Figure $5 b$ ).

We next investigated possible relationships between smRNA accumulation and DNA methylation. We found that almost all of the altered $\mathrm{CHH}$ methylation was associated with changes in smRNA clusters. For example, the increased $\mathrm{CHH}$ methylation of the Pong copy located on chromosome 2 was associated with a moderate increase in smRNA accumulation, whereas the slight decrease in $\mathrm{CHH}$ methylation in the flanking region and the increase in $\mathrm{CHH}$ methylation in the gene body region of the Pong copy located on chromosome 9 were associated with moderate decrease and increase in smRNA accumulations, respectively (Additional file $5 \mathrm{c}$ ). These positive correlations of smRNA accumulation and $\mathrm{CHH}$ methylation suggests that OsRDR1 plays a role in the de novo $\mathrm{CHH}$ methylation in a subset of genomic loci in rice, probably by affecting the production/accumulation of smRNAs required for RdDM, as shown in Arabidopsis $[20,21]$. The locus-specificity of methylation changes or the two analyzed TEs indicated that their methylation patterns were determined by either or both the flanking sequences and the local chromatin environment, an issue which warrants further investigations.

\section{Phenotypes in Osrdr1 under normal and abiotic stress conditions}

It is known that various stress conditions may produce protracted effects on genome stability, leading to transgenerational changes in genome structure, which are proposed to have been initiated by epigenetic mechanisms [31-37]. We were therefore interested to know if OsRDR1 may play a role in stress response in rice. We quantified phenotypes between Osrdr1 and WT plants under normal and several short-term abiotic stress conditions (see Materials and Methods), which included treatments with salt, heavy metals $\mathrm{Cu}^{2+}$ and $\mathrm{Hg}^{2+}$, and 


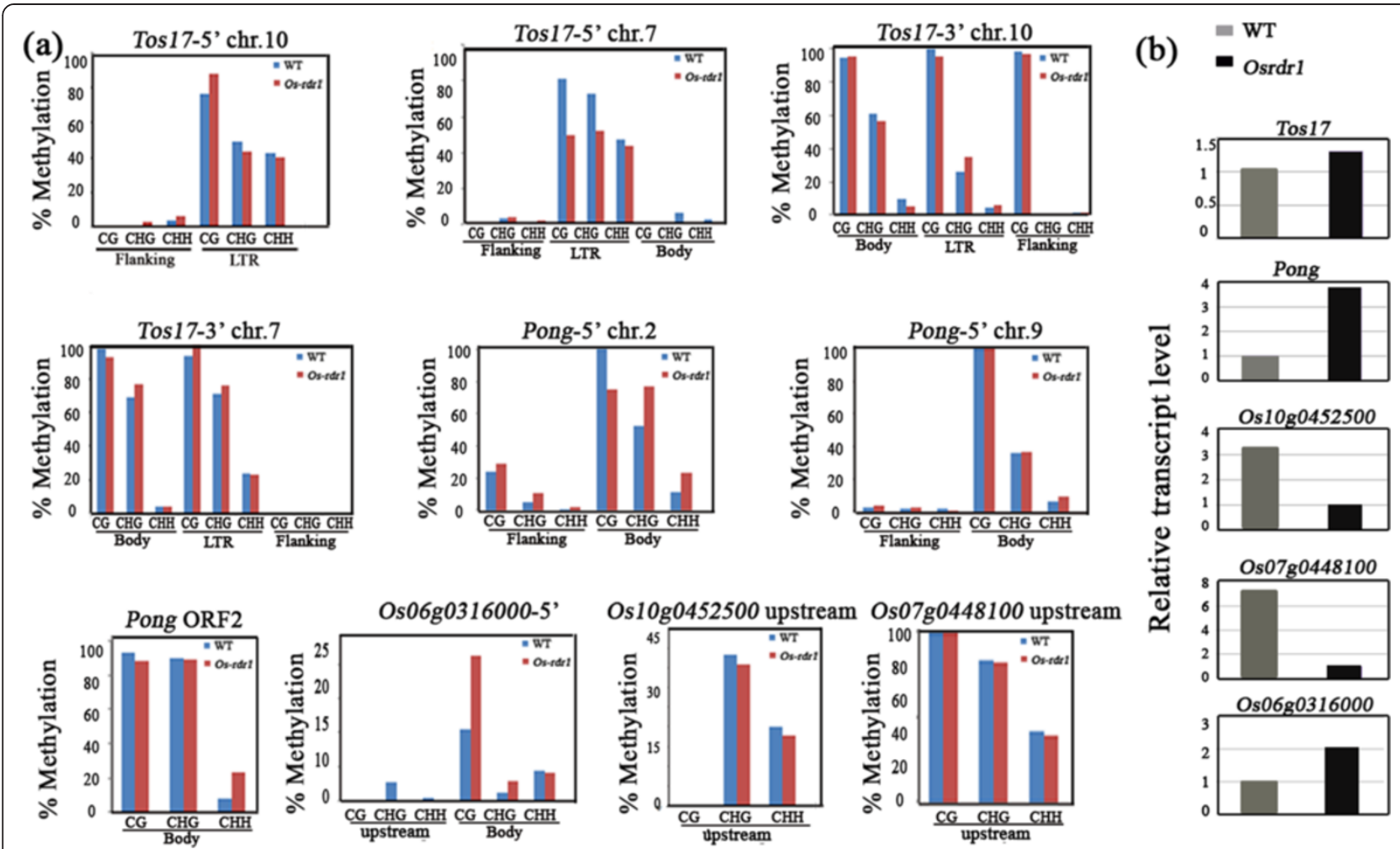

Figure 5 Effects of null mutation of OsRDR1 on locus-specific alterations of DNA methylation in rice. (a) Alteration in DNA methylation between Osrdr1 and WT in the three cytosine sequence contexts, CG, CHG and CHH, based on bisulphite sequencing of 10 genomic loci from two transposable elements (TEs), Tos 17 (four regions) and Pong (three regions) and three genes (one region each). (b) Expression differences of the TEs and genes between Osrdr1 and WT based on qRT-PCR analysis.

overdose nitric oxide (NO). The results showed that no phenotypic difference was found between Osrdr1 and WT under normal condition, but significant ephemeral phenotypic differences between the two genotypes emerged in some of the different stress conditions (Figure 6). Specifically, (1) seedlings of Osrdr1 were more sensitive than WT to salt and overdose NO treatments, as being reflected by reduced plant height, root length and biomass at the seedling stage, with the difference in plant height and root length being persisted to the heading stage after removal of the stresses; (2) Seedlings of Osrdr1 showed increased tolerance to heavy metal $\mathrm{Cu}^{2+} / \mathrm{Hg}^{2+}$ as indicated by increased root length, but upon removal of the stresses the differences were gradually attenuated and completely disappeared at the heading stage; (3) When both unstressed and the transiently-stressed plants of the mutant and WT were grown to maturity, no difference in plant height, tiller number, panicle and kernel traits was observed between the two genotypes. Collectively, our results suggest that $O s R D R 1$ has a potential function in stress response in rice, but the effects are contingent with presence of stresses without exerting protracted influence when the stresses are removed.

\section{Discussion}

RNA silencing pathways have been well characterized in the dicot model plant Arabidopsis but remain poorly studied in other plants like monocots to which many major crops belong. Utilizing a retransposon Tos 17 insertion mutant of OsRDR1 in rice, we performed genome-wide analysis to unveil the function of OsRDR1, a component recently shown in Arabidopsis to be involved in noncanonical, 21 nt siRNA-directed RdDM pathway [20,21], on expression of endogenous genes. By deep sequencing of smRNAs and microarray analysis of gene expression in the Osrdr1 mutant and its sibling WT, we showed that the expression of $>1,000$ genes were significantly changed in Osrdr1 relative to WT, suggesting that OsRDR1 plays a role in genome-wide gene regulation in rice. In addition, the Osrdr1 mutant showed regional alterations in smRNA accumulation and/or titration across the rice genome, and at least some of which are associated with locus-specific alteration of DNA methylation.

Among the differentially accumulated smRNAs, many were miRNAs both previously known and newly identified in this study. Expression changes in many of these miRNAs are associated with changes in their target gene 


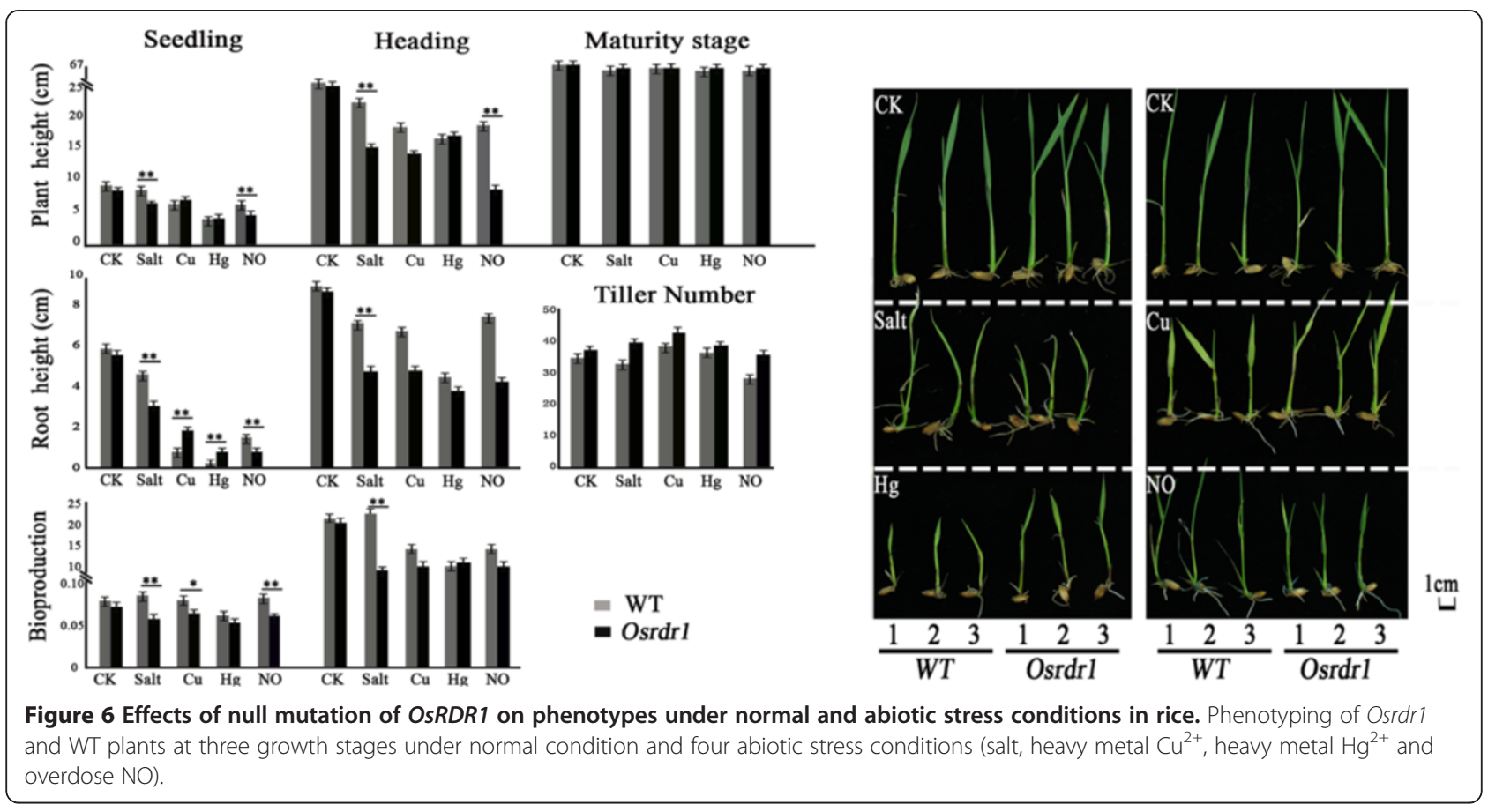

expression, which could partly be responsible for the gene expression changes observed in the Osrdr1 mutant relative to WT. The mechanism by which mutation of OsRDR1 caused changes in miRNA expression in rice was not clear. It was found in Arabidopsis that none of the RDRs has a direct role in miRNA biogenesis [2,38]. However, RDRs could impact miRNA accumulation indirectly by either affecting miRNA precursor gene expression through the TGS or PTGS pathways $[1,2,38]$ or generating dsRNAs that compete for DCL1 function that is required for miRNA biogenesis [1,2].

Our results suggested that OsRDR1 might play a role in maintaining the intrinsic locus-specific DNA methylation patterns, as its mutation caused alteration of methylation at some of the loci we analyzed. In particular, the changes in $\mathrm{CHH}$ methylation, which are indicative of $d e$ novo methylation by the RdDM pathway, showed correlation with changes in smRNA accumulation. In the respect of reduced $\mathrm{CHH}$ methylation and concomitant reduced smRNA accumulation, OsRDR1 may functionally resemble the Arabidopsis $R D R 1$ and plays a role in the 21 nt siRNA-dependent non-canonical RdDM pathway $[20,21]$. However, some of the analyzed loci showed increased $\mathrm{CHH}$ methylation that is associated with increased smRNA accumulation in Osrdr1. We should caution that because we analyzed only 10 loci, the results observed may not be extrapolated to global scale. In Arabidopsis, it was documented that mutation of RDR1 resulted in near complete loss of methylated cytosine of all three sequence contexts (CG, CHG and $\mathrm{CHH}$ ) within the 4,949 CHH hypomethylation DMRs (differentially methylated regions) between $d r m 1 / 2$ and WT [20]. Therefore, genome-wide methylation analysis (methylome) of the Osrdr1 mutant will be required to confirm whether OsRDR1 plays a similar role globally in rice.

Previous studies in Arabidopsis and Nicotiana have defined an established role of RDR1 in plant virus responses $[24,26,27]$. We showed here that Osrdr1 exhibited no phenotypic differences from its sibling WT plants under normal growing condition, but displayed ephemeral phenotypic fluctuations contingent with presence of several abiotic stress conditions. This observation, together with the enriched GO categories including those involved in metabolic process of the differentially expressed genes between Osrdr1 and WT (Figure 2b), suggest that the effects of altered gene expression due to OsRDR1 mutation has been largely canalized under normal condition but can be released by certain abiotic stress conditions [39], an issue that merits further investigations. Regardless, our results suggest that, apart from its established role in the production and amplification of exogenous, virusderived siRNAs (vsiRNAs) in infected plants [26,27], the rice $R D R 1$ homolog $(O s R D R 1)$ might also play a role in certain abiotic stress responses, which however may not involve stable epigenetic changes in this respect. In this regard, it should be emphasized again that the genome-wide analyses of both smRNA profiles and gene expression in Osrdr1 were conducted on plants grown under normal conditions. Therefore, further studies are needed to conduct the analyses in plants of the mutant and WT under both short- and long-term stress conditions. It would also be interesting to analyze the progeny of stress-treated 
Osrdr1 plants to investigate if OsRDR1 is involved in transgenerational inheritance of stress-induced epigenetic changes, if they occurred.

\section{Conclusions}

How RDR1 affects global gene expression and smRNA profiles have not been previously investigated in any plant species. By analyzing a null mutant of the rice $R D R 1$ gene (OsRDR1), we showed that expression of more than 1,000 endogenous genes of diverse gene ontology (GO) categories were significantly altered in the mutant, indicating a functional role of OsRDR1 in regulating endogenous gene expression in rice. By smRNA deep-sequencing, we found that extensive alteration in smRNA clusters occurred across each of the 12 rice chromosomes in the mutant, indicating a role of OsRDR1 in smRNA biogenesis and/or titration in rice. We also found that at least some of the gene expression changes are correlated with differences in miRNAs. We further showed that changes in smRNAs can be concomitant with locus-specific alteration of cytosine methylation primarily of the $\mathrm{CHH}$ contexts, thus linking OsRDR1 to DNA methylation in rice. Finally, we showed that whereas no apparent phenotypic abnormality was associated with loss of function of OsRDR1, ephemeral phenotypic fluctuations could be generated by various short-term abiotic stress conditions as a result of OsRDR1 mutation, suggesting a role of $O s R D R 1$ in plant abiotic stress response.

\section{Methods}

\section{Plant materials}

Based on information about the rice retrotransposon Tos17 insertion lines (http://tos.nias.affrc.go.jp/), we obtained a line (\#RDR704) of rice cultivar Hitomebore with Tos 17 being inserted into the second exon of OsRDR1 in a heterozygous state (accession \# H0643). According to BlastN search at the NCBI website (http://blast.ncbi.nlm. nih.gov/Blast.cgi), we found that rice contains a single copy of the insert gene (OsRDR1). The three OsRDR1 genotypes, WT (RDR1/RDR1), heterozygous (RDR1/rdr1) and homozygous mutant $(r d r 1 / r d r 1)$ were identified by two pairs of specific primers (Figure 1a). Specifically, WT was identified by a pair of primers anchored within the OsRDR1 gene but flanking the Tos17 insertion site; homozygous mutant was identified by a pair primers with on anchored to the OsRDR1 gene and the other one targeting to the terminal of Tos17; and the heterozygote was identified by combinations of both types of primers. The heterozygotes of OsRDR1(+/-) were selfed for five successive generations, and in each of the first four generation (S1-S4) only heterozygous individuals were selected based on PCR identification. At the last generation (S5), the newly segregated homozygous mutant, i.e., OsRDR1-/(designated as $O s r d r 1$ ), and its sibling wild type (WT), i.e.,
OsRDR1+/+, were selected and propagated for an additional generation to have sufficient seeds for this study. In this way, the mutant and WT should be genetically identical except for the locus in concern, i.e., OsRDR1. Seeds of the two genotypes were thoroughly washed with distilled water and then germinated in the dark in Petri dishes containing distilled water at $28^{\circ} \mathrm{C}$. After a 2-day incubation, germinated seeds were transferred to a greenhouse at $26^{\circ} \mathrm{C}$ under $16 \mathrm{~h} / 8 \mathrm{~h}$ light/dark regime for the four kinds of abiotic stress treatments: $0.15 \mathrm{mMol} / \mathrm{L} \mathrm{NaCl}$ (salt), $0.25 \mathrm{mMol} / \mathrm{L} \mathrm{CuSO}_{4}$ (heavy metal $\mathrm{Cu}^{2+}$ ), $0.25 \mathrm{mMol} / \mathrm{L}$ $\mathrm{HgCl}_{2}$ (heavy metal $\mathrm{Hg}^{2+}$ ), $1 \mathrm{mMol} / \mathrm{L}$ Sodium nitroferricyanide(III) dehydrate (SNP, for NO stress) in Hoagland nutrient solution for 7- day. Mock controls (CK) were grown in parallel. Then, all seedling plants were transplanted to normal paddy field. Plants were surveyed at appropriate growth and developmental stages, seedling, heading and maturity.

Genomic DNA was isolated from seedlings of Osrdr1 and WT at the same developmental stage using a modified CTAB method. Total RNA was isolated from the same seedlings with the Trizol Reagent (Invitrogen) according to the manufacturer's instructions. The RNA was then treated with RNase-free DNase I (Invitrogen) to eliminate possible genomic DNA contamination before being reverse transcribed with the SuperScript RNase H- Reverse Transcriptase (Invitrogen).

\section{SmRNA library construction and sequencing}

Total RNA was prepared for smRNA sequencing based on the Illumina Sample Preparation Protocol. The samples were quantified and equalized so that equivalent amounts of RNA from Osrdr1 and WT were analyzed. In brief, total RNA was purified by electrophoretic separation on a $15 \%$ TBE-urea denaturing PAGE gel and smRNA regions corresponding to the 15-30 nucleotide bands in the marker lane were excised and recovered. The 15-30 nt smRNAs were 5' and 3' RNA adapter-ligated by T4 RNA ligase and at each step length validated and purified by urea PAGE gel electrophoretic separation. The adapter-ligated smRNA was subsequently transcribed into cDNA by Super- Script II reverse transcriptase (Invitrogen) and PCR amplified, using primers that anneal to the ends of the adapters. The amplified cDNA, too, was purified and recovered. The final quality of the library was ensured by validation of the size, purity and concentration using an Agilent Technologies 2100 Bioanalyzer. The two constructed cDNA libraries subsequently underwent Solexa/Illumina's proprietary flowcell cluster generation and bridge amplification.

\section{Analysis of smRNA clusters}

SmRNA reads of 18-26 nt in size were counted within every sliding $100 \mathrm{bp}$ window along the rice genome. The reads were normalized to RPM (reads per million), and 
comparison was then made between Osrdr1 and WT plants using the median RPM values, which are denoted as $\mathrm{X}$ for Osrdr1 and $\mathrm{Y}$ for WT plants. The fold value was calculated by the formula $\log _{2} \mathrm{X}-\log _{2} \mathrm{Y}=\log _{2}(\mathrm{X} / \mathrm{Y})$.

\section{Affymetrix GeneChip ${ }^{\oplus}$ Rice Genome Array}

The microarray transcriptional profiling was performed by the Affymetrix, Inc. in the Gene Company Ltd. (Shanghai, China), using procedures described in the GeneChip ${ }^{\circ}$ Expression Analysis Technical Manual.

\section{Real-time quantitative (q) reverse transcriptase (RT)-PCR analysis}

The qRT-PCR experiments were performed using SYBR Premix Ex Taq (TOYOBO) according to the manufacturer's instruction on a Roche LightCycler480 apparatus (Roche Inc.). The primers for amplifying the 18 studied genes were designed using the Primer 5 software and listed in Additional file 6. Primers for qRT-PCR analysis of transposase genes were described in a previous report [40]. Expression of a rice $\beta$-actin gene, $e E F$ gene and $U B Q 5$ gene were used as internal control with the primer pairs of5'-atgccattctccgtctt and 5'-gctcctgctcgtagtc; 5'-tttc actcttggtgtgaagcagat and 5'-gacttccttcacgatttcatcgtaa; 5'accacttcgaccgccactact and 5 -acgcctaagcctgctggtt, respectively. Conditions of RT-qPCR were as reported [40].

\section{Hemi-nested RT-PCR for detecting miRNA expression}

Four miRNA-specific oligonucleotide primers were designed according to a previous report $[41,42]$ to reverse transcribe the specific miRNAs, and four pairs of specific primers corresponding to the RT primers were designed to amplify the cDNA (Additional file 6). RT-PCR was performed as previously described $[41,42]$.

\section{Bisulphite sequencing}

DNA $(\sim 1 \mu \mathrm{g})$ from each plant was treated with bisulphite using the EZ DNA Methylation-Gold Kit and amplified using specific primers (Additional file 6). Bisulphite PCR product was cloned into vector NTI and positive clones were sent for sequencing. The results were analyzed using analysis software on the website http://katahdin.mssm. edu/kismeth based on a previous report [43].

\section{Additional files}

Additional file 1: Figure S1. Chromosomal distribution of differential smRNA clusters between Osrdr1 and WT for a selected subset smRNAs in the size ranges of 20-24 nt. Where $x$ axis is the length of chromosome (Per $100 \mathrm{bp}$ window) and $y$ axis is the value of different RPMs (log value, base 2). The vertical blue lines denote centromeric regions in each chromosome.

Additional file 2: Table S1. List of expression of known miRNAs between Osrdrl and wide-type.
Additional file 3: Figure S2. Putative novel miRNAs identified from Osrdr1 and wide-type. (a) The sequences, expression status including read counts, and genomic locations of the novel miRNAs. $5 p$, the mature miRNA sequence resides in $5^{\prime}$ half of the predicted stem-loop structure; $3 p$, the mature miRNA sequence resides in $3^{\prime}$ half of the predicted stem-loop structure. mfe, minimum free energy. (b) The predicted stem-loop structure of precursor RNA of the novel miRNAs. The mature miRNA sequence inside the stem-loop is indicated by a red line, and the $5^{\prime}$ to $3^{\prime}$ direction of a miRNA is indicated by an arrowhead.

Additional file 4: Figure S3. (a) Correlation of expression of known miRNAs and their targets. (b) Pairwise comparison between expression levels of known miRNAs and their targets. Yellow and blue columns represent target and miRNA expression levels, respectively. $Y$-axis indicates the values of $\log _{2}$ fold change.

Additional file 5: Figure S4. Regional association between smRNA clusters and DNA methylation for each of the 10 assayed genomic loci from two transposable elements (TEs), Tos 17 (four regions) and Pong (three regions) and three genes (one region each). The red, blue and green circles denote for $\mathrm{CG}, \mathrm{CHG}$ and $\mathrm{CHH}$ sequence contexts, respectively, wherein the filled ones are methylated and empty ones are unmethylated.

Additional file 6: Table S2. Primers for qRT-PCR assay of 18 genes, bisulfite sequencing of 10 loci and semi-nested qRT-PCR analysis of four miRNAs.

\section{Competing interests}

The authors declare that they have no competing interests.

\section{Authors' contributions}

NW and DZ carried out major parts of the experiments, analyzed the data and drafted the manuscript. ZHW, HWX, JM, HW, WH, YL, XYL, CYZ, OXF and $\mathrm{NL}$ participated in all the experiments. BL and MBW designed the work and finalized the manuscript. All authors read and approved the final manuscript.

\section{Acknowledgements}

This study was supported by the National Natural Science Foundation of China (30990243, 31200198), by the State Key Basic Research and Development Plan of China (2013CBA01404), the Jilin Provincial Research Foundation for Basic Research, China (201201097, 201205048), and a Special Foundation for Young Scientists of Jilin Agricultural University (201201).

\section{Author details}

'Key Laboratory of Molecular Epigenetics of Ministry of Education (MOE), Northeast Normal University, Changchun 130024, China. ${ }^{2}$ Faculty of Agronomy, Jilin Agricultural University, Changchun 130118, China. ${ }^{3} \mathrm{Jilin}$ Academy of Agricultural Sciences, Changchun 130033, China. ${ }^{4}$ School of Food Production Technology and Biotechnology, Changchun Vocational Institute of Technology, Changchun, China. ${ }^{5}$ Commonwealth Scientific and Industrial Research Organisation Plant Industry, Canberra, Australian Capital Territory 2601, Australia.

Received: 24 January 2014 Accepted: 3 June 2014

Published: 30 June 2014

\section{References}

1. Baulcombe D: RNA silencing in plants. Nature 2004, 431:356-363.

2. Eamens $A$, Wang $M B$, Smith NA, Waterhouse PM: RNA silencing in plants: yesterday, today, and tomorrow. Plant Physiol 2008, 147(2):456-468.

3. Zamore PD: RNA interference: listening to the sound of silence. Nat Struct Biol 2001, 8:746-750.

4. Tang G: siRNA and miRNA: an insight into RISCs. Trends Biochem Sci 2005, 30(2):106-114

5. Taylor RS, Tarver JE, Hiscock SJ, Donoghue PC: Evolutionary history of plant microRNAs. Trends Plant Sci 2014, 19(3):175-182.

6. Wang MB, Masuta C, Smith NA, Shimura H: RNA silencing and plant viral diseases. Mol Plant Microbe Interact 2012, 25(10):1275-1285.

7. Haag JR, Pikaard CS: Multisubunit RNA polymerases IV and V: purveyors of non-coding RNA for plant gene silencing. Nat Rev Mol Cell Biol 2011, 12:483-492. 
8. Matzke M, Kanno T, Daxinger L, Huettel B, Matzke AJ: RNA-mediated chromatin-based silencing in plants. Curr Opin Cell Biol 2009, 21(3):367-376

9. Zhang $\mathrm{H}$, Zhu JK: RNA-directed DNA methylation. Curr Opin Plant Biol 2011, 14:142-147.

10. Sun F, Guo W, Du J, Ni Z, Sun Q, Yao Y: Widespread, abundant, and diverse TE-associated siRNAs in developing wheat grain. Gene 2013, 522(1):1-7.

11. Wassenegger M, Krczal G: Nomenclature and functions of RNA-directed RNA polymerases. Trends Plant Sci 2006, 11(3):142-151.

12. Song X, Wang D, Ma L, Chen Z, Li P, Cui X, Liu C, Cao S, Chu C, Tao Y, Cao $X$ : Rice RNA-dependent RNA polymerase 6 acts in small RNA biogenesis and spikelet development. Plant J 2012, 71(3):378-389.

13. Vazquez F, Vaucheret $H$, Rajagopalan R, Lepers C, Gasciolli V, Mallory AC, Hilbert $J$, Bartel DP, Crete P: Endogenous trans-acting siRNAs regulate the accumulation of Arabidopsis mRNAs. Mol Cell 2004, 16(1):69-79.

14. Shivaprasad PV, Rajeswaran R, Blevins T, Schoelz J, Meins F Jr, Hohn T, Pooggin MM: The CaMV transactivator/viroplasmin interferes with RDR6-dependent trans-acting and secondary siRNA pathways in Arabidopsis. Nucleic Acids Res 2008, 36(18):5896-5909.

15. Vaistij FE, Jones $\mathrm{L}$ : Compromised virus-induced gene silencing in RDR6-deficient plants. Plant Physiol 2009, 149(3):1399-1407.

16. Zhu QH, Stephen S, Taylor J, Helliwell CA, Wang MB: Long noncoding RNAs responsive to Fusarium oxysporum infection in Arabidopsis thaliana. New Phytol 2014, 201(2):574-584.

17. Ding SW, Li H, Lu R, Li F, Li WX: RNA silencing: a conserved antiviral immunity of plants and animals. Virus Res 2004, 102(1):109-115.

18. Liao YW, Sun ZH, Zhou YH, Shi K, Li X, Zhang GQ, Xia XJ, Chen ZX, Yu JQ: The role of hydrogen peroxide and nitric oxide in the induction of plant-encoded RNA-dependent RNA polymerase 1 in the basal defense against Tobacco mosaic virus. PLoS One 2013, 8(9):e76090.

19. Rakhshandehroo F, Takeshita M, Squires J, Palukaitis P: The influence of RNA-dependent RNA polymerase 1 on potato virus $Y$ infection and on other antiviral response genes. Mol Plant Microbe Interact 2009, 22(10):1312-1318.

20. Stroud H, Greenberg MV, Feng S, Bernatavichute $Y$, Jacobsen SE: Comprehensive analysis of silencing mutants reveals complex regulation of the Arabidopsis methylome. Cell 2013, 152(1-2):352-364.

21. Pontier D, Picart C, Roudier F, Garcia D, Lahmy S, Azevedo J, Alart E, Laudie M, Karlowski WM, Cooke R, Colot V, Voinnet O, Lagrange T: NERD, a plant-specific GW protein, defines an additional RNAi-dependent chromatin-based pathway in Arabidopsis. Mol Cell 2012, 48(1):121-132

22. Chen H, Kobayashi K, Miyao A, Hirochika H, Yamaoka N, Nishiguchi M: Both OsRecQ1 and OsRDR1 are required for the production of small RNA in response to DNA-damage in rice. PLoS One 2013, 8(1):e55252.

23. Chen H, Tamai A, Mori M, Ugaki M, Tanaka Y, Samadder P, Miyao A, Hirochika H, Yamaoka N, Nishiguchi M: Analysis of rice RNA-dependent RNA polymerase 1 (OsRDR1) in virus-mediated RNA silencing after particle bombardment. J Gen Plant Pahtol 2010, 76(3):152-160.

24. Ying $X B$, Dong $L$, Zhu $H$, Duan CG, Du QS, Lv DQ, Fang YY, Garcia JA, Fang RX, Guo HS: RNA-dependent RNA polymerase 1 from Nicotiana tabacum suppresses RNA silencing and enhances viral infection in Nicotiana benthamiana. Plant Cell 2010, 22(4):1358-1372.

25. Hirochika H, Sugimoto K, Otsuki Y, Tsugawa H, Kanda M: Retrotransposons of rice involved in mutations induced by tissue culture. Proc Natl Acad SCl US A 1996, 93(15):7783-7788.

26. Donaire L, Barajas D, Martinez-Garcia B, Martinez-Priego L, Pagan I, Llave C. Structural and genetic requirements for the biogenesis of tobacco rattle virus-derived small interfering RNAs. J Virol 2008, 82:5167-5177.

27. Wang XB, Wu Q, Ito T, Cillo F, Li WX, Chen X, Yu JL, Ding SW: RNAimediated viral immunity requires amplification of virus-derived siRNAs in Arabidopsis thaliana. Proc Natl Acad Sci U S A 2010, 107:484-489.

28. Sunkar R, Zhou X, Zheng Y, Zhang W, Zhu JK: Identification of novel and candidate miRNAs in rice by high throughput sequencing. BMC Plant Biol 2008, 8:25.

29. Zhu QH, Spriggs A, Matthew L, Fan L, Kennedy G, Gubler F, Helliwell C: A diverse set of microRNAs and microRNA-like small RNAs in developing rice grains. Genome Res 2008, 18(9):1456-1465.

30. Lu C, Jeong DH, Kulkarni K, Pillay M, Nobuta K, German R, Thatcher SR, Maher C, Zhang L, Ware D, Liu B, Cao X, Meryers B, Green P: Genome-wide analysis for discovery of rice microRNAs reveals natural antisense
microRNAs (nat-miRNAs). Proc Natl Acad Sci U S A 2008, 105(12):4951-4956.

31. Chinnusamy $\mathrm{V}$, Zhu JK: Epigenetic regulation of stress responses in plants. Curr Opin Plant Biol 2009, 12(2):133-139.

32. Karan R, DeLeon T, Biradar H, Subudhi PK: Salt stress induced variation in DNA methylation pattern and its influence on gene expression in contrasting rice genotypes. PLoS One 2012, 7(6):e40203.

33. Kim JM, To TK, Ishida J, Matsui A, Kimura H, Seki M: Transition of chromatin status during the process of recovery from drought stress in Arabidopsis thaliana. Plant Cell Physiol 2012, 53(5):847-856.

34. Kou HP, Li Y, Song XX, Ou XF, Xing SC, Ma J, Von Wettstein D, Liu B: Heritable alteration in DNA methylation induced by nitrogen-deficiency stress accompanies enhanced tolerance by progenies to the stress in rice (Oryza sativa L.). J Plant Physiol 2011, 168(14):1685-1693.

35. Xin M, Wang $Y$, Yao $Y$, Xie C, Peng $H$, Ni Z, Sun Q: Diverse set of microRNAs are responsive to powdery mildew infection and heat stress in wheat (Triticum aestivum L.). BMC Plant Biol 2010, 10:123.

36. Ou X, Zhang Y, Xu C, Lin X, Zang Q, Zhuang T, Jiang L, von Wettstein D, Liu $B$ : Transgenerational inheritance of modified DNA methylation patterns and enhanced tolerance induced by heavy metal stress in rice (Oryza sativa L.). PLoS One 2012, 7:e41143.

37. Zhu $Y$, Dong A, Shen $\mathrm{WH}$ : Histone variants and chromatin assembly in plant abiotic stress responses. Biochim Biophys Acta 2012, 1819(3-4):343-348.

38. Bartel DP: MicroRNAs: genomics, biogenesis, mechanism, and function. Cell 2004, 116(2):281-297.

39. Hermisson J, Wagner GP: The population genetic theory of hidden variation and genetic robustness. Genetics 2004, 168(4):2271-2284.

40. Wang N, Wang H, Zhang D, Wu Y, Ou X, Liu S, Dong Z, Liu B: Transpositional reactivation of the Dart transposon family in rice lines derived from introgressive hybridization with Zizania latifolia. BMC Plant Biol 2010, 10:190.41.

41. Chen C, Ridzon DA, Broomer AJ, Zhou Z, Lee DH, Nguyen JT, Barbisin M, Xu NL, Mahuvakar VR, Andersen MR, Lao K, Livak K, Guegler K: Real-time quantification of microRNAs by stem-loop RT-PCR. Nucleic Acids Res 2005, 33(20):e179.

42. Wan G, Lim QE, Too HP: High-performance quantification of mature microRNAs by real-time RT-PCR using deoxyuridine-incorporated oligonucleotides and hemi-nested primers. RNA 2010, 16(7):1436-1445.

43. Ngezahayo F, Xu C, Wang H, Jiang L, Pang J, Liu B: Tissue culture-induced transpositional activity of mPing is correlated with cytosine methylation in rice. BMC Plant Biol 2009, 9:91.

doi:10.1186/1471-2229-14-177

Cite this article as: Wang et al:: Mutation of the RDR1 gene caused genome-wide changes in gene expression, regional variation in small RNA clusters and localized alteration in DNA methylation in rice. BMC Plant Biology 2014 14:177.

\section{Submit your next manuscript to BioMed Central and take full advantage of:}

- Convenient online submission

- Thorough peer review

- No space constraints or color figure charges

- Immediate publication on acceptance

- Inclusion in PubMed, CAS, Scopus and Google Scholar

- Research which is freely available for redistribution 